\title{
Mean platelet volume in diagnosis of gestational diabetes
}

\author{
Erçin Erdem Çelikel, Sefa Kurt, Emrah Töz*, Azra Arıcı, Tutku Gürbüz, Abdullah Taşyurt \\ Department of Gynecology and Obstetrics, Izmir Ege Gynecology and Obstetrics Teaching and Research Hospital, İzmir, Turkey
}

\section{ARTICLE INFO}

\section{Article History}

$\begin{array}{ll}\text { Received } & 26 / 06 / 2013 \\ \text { Accepted } & 15 / 08 / 2013\end{array}$

\section{* Correspondence to:}

Emrah Töz

Department of Gynecology and Obstetrics,

Izmir Ege Teaching and Research Hospital,

Izmir, Turkey

e-mail: emrahtoz79@gmail.com

\section{Keywords:}

Diagnostic test

Gestational diabetes mellitus

Impaired glucose tolerance

Mean platelet volume

\author{
ABSTRACT
}

\begin{abstract}
Gestational diabetes mellitus (GDM) prevalence is within range of 1-14\%, varying relative to the countries and ethnic groups. By many studies, mean platelet volume (MPV) has been shown to be higher in GDM and Type II diabetes mellitus patients. Our aim is to evaluate the sensitivity and specificity of MPV in GDM screening. The study population was consisted of pregnant women aged above 18 years with a singleton pregnancy at 24 to 28 weeks of gestation. Three hundred patients complying with the study criteria were split into 3 groups as follows: Group 1,219 pregnant patients with normal 50g oral glucose tolerance test (OGTT) result; Group 2, 23 pregnant patients diagnosed with impaired glucose tolerance based on the OGTT result; and Group 3, 58 pregnant patients diagnosed with GDM. MPV measurement was started with collecting $2 \mathrm{ml}$ blood samples into K3 Etilendiamintetraasetikasit (EDTA) tubes from each patient. The statistical analyses were done with statistical package for the social sciences (SPSS) 20.0 package program. In our study, the optimal cut-off MPV value for GDM prediction was $>8.8 \mathrm{fL}$. The specificity and negative predictive value of MPV at $>8,8 \mathrm{fL}$ were $75.9 \%$ and $38.9 \%$, respectively. GDM patients may exhibit significant changes in MPV and hemoglobin A1c (HbA1c) values. Future randomized studies will be helpful in developing a MPV-related diagnostic test, adjusting the treatment, predicting the complications, and taking preventive measures in GDM patients.
\end{abstract}

J. Exp. Clin. Med., 2013; 30:291-294

(C) 2013 OMU

\section{Introduction}

Gestational diabetes mellitus (GDM) and Impaired Glucose Tolerance (IGT) are the most common metabolic disorders seen during pregnancy which have importance because they may lead to fetal and maternal complications. GDM prevalence occurs within range of $1-14 \%$, varying relative to the countries and ethnic groups. The developing risk of Type II DM within 5-16 years in women with GDM is $17-63 \%$ (Mazze and Krogh, 1992, Harris et al., 1997).

By many studies, mean platelet volume (MPV) has been shown to be higher in GDM and Type II diabetes mellitus patients than in the normal population (Hekimsoy et al., 2004; Papanas et al. 2004). Two different theories are discussed for the underlying mechanism of elevated platelet volume. One of these theories is based on osmotic swelling due to increased blood glucose concentration and elevated levels of some glucose metabolites. Another possible mechanism focuses on increased platelet turnover associated with platelet hyperactivity. In diabetic patients, the amount of intracellu- lar free calcium and calcium release from platelets increase, thereby leading to the activation of arachidonic pathway which increases the formation of thromboxane A2 (TxA2). TxA2 activity, in turn, causes thrombopoietic stress associated with platelet hyperactivity and raised turnover, giving rise to megakaryocytic growth.

Granules of larger platelets secrete higher amount of serotonin and $\beta$ tromboglobulin, and produce higher volume of TxA 2 (Bath and Butterworth, 1996). Furthermore, they contain higher amounts of similar adhesion molecules such as P-selectin and glycoprotein IIb/IIIa (Jagroop and Mikhailidis, 2001). Large platelets have higher activity and are more prone to adhesion and aggregation (Hekimsoy et al., 2004; Park et al., 2002). Therefore, MPV alone is recognized as a marker of the platelet activation process (Bath and Butterworth, 1996).

Our primary end point in this study is to investigate the relationship of MPV levels with blood sugar and hemoglobin concentrations, whereas our second end point is to evaluate the sensitivity and specificity of MPV in GDM screening. 


\section{Materials and methods}

This study was conducted at the Izmir Ege Gynecology and Obstetrics Teaching and Research Hospital between January 2012 and June 2012. The study population was consisted of pregnant women aged above 18 years with a singleton pregnancy at 24 to 28 weeks of gestation. Exclusion criteria were as follows: Pregestational diabetes; multiple pregnancy; hemoglobinopathy; hematologic, hypertensive, or chronic inflammatory disease; an endocrine disease that may cause diabetes, and smoking cigarette. Informed consent was obtained from all women prior to inclusion in the study, which was approved by the Local Human Investigation Committee.

Subsequent to a fasting period of 8-14 hours, blood was collected from the pregnant women and 1-hour oral glucose tolerance test (OGTT) was performed with standard $50 \mathrm{~g}$ glucose (Glucose, Dextrose Monohydrat $50 \mathrm{~g}$ ). Glucose measurement was carried out from venous blood using the enzymatic method. The cut-off value for 1-hour glucose tolerance test after $50 \mathrm{~g}$ glucose load was defined as $140 \mathrm{mg} / \mathrm{dL}$. The glucose levels above $180 \mathrm{mg} / \mathrm{dL}$ were recognized as GDM. The women with a glucose value between $140-180 \mathrm{mg} / \mathrm{dL}$ underwent 100g 3-hour OGTT test. GDM diagnosis was based on Carpenter and Coustan criteria $(\mathrm{C} \& \mathrm{C})$, as exceeding two of the threshold values for OGTT. In cases where only one threshold was exceeded, the diagnosis was impaired glucose tolerance (Table 1).

Table 1. Threshold values used in 100g OGTT test in GDM
diagnosis based on the Carpenter and Coustan (C\&C)
criteria

GDM: Gestational diabetes mellitus; OGTT: Oral glucose tolerance test

MPV measurement was started with collecting $2 \mathrm{ml}$ blood samples into K3 EDTA tubes from each patient. The analysis was performed by Beckman Coulter device within 2 hours from the collection, while HbAlc was studied with immunoturbidimetric inhibition method.

The statistical analyses were done with SPSS 20.0 package program. The distribution of the data was tested with Kolmogorov-Smirnov. The variables were analyzed with ANOVA (Tukey test), whereas covariates were studied with MANCOVA, and correlation analysis was carried out with Pearson's correlation analysis. $\mathrm{p}<0.05$ is considered to be statistically significant.

\section{Results}

A total of 300 patients complying with the study criteria were split into 3 groups as follows: The Group 1, 219 preg- nant patients with normal 50g OGTT result; the Group 2, 23 pregnant patients diagnosed with impaired glucose tolerance based on the OGTT result; and the Group 3, 58 pregnant patients diagnosed with GDM.

The Group 1 had significantly lower mean age than Groups 2 and $3(p<0.05)$, whereas the Group 2 showed significantly higher gestational age as compared to the Groups 1 and $3(\mathrm{p}<0.05)$ (Table 2).

The hemoglobin value showed a homogenous distribution among the groups, while MPV value was significantly higher in the Group 3 than in the Groups 1 and 2, and $\mathrm{HbAlc}$ value was significantly higher in the Groups 2 and 3 than in Group $1(\mathrm{p}<0.05)$ (Table 3$)$.

\begin{tabular}{|c|c|c|c|c|}
\hline & Group 1 & Group 2 & Group 3 & \\
\hline & Mean \pm s.d & Mean \pm s.d & Mean \pm s.d & $P$ \\
\hline Age & $25.75 \pm 5.20$ & $29.22 \pm 4.72$ & $30.12 \pm 4.68$ & 0.000 \\
\hline Gestational age & $25.42 \pm 1.40$ & $26.57 \pm 2.21$ & $25.50 \pm 1.31$ & 0.017 \\
\hline
\end{tabular}

The comparison of the three groups with regard to MPV, $\mathrm{HbA1c}$, and fasting blood glucose (FBG) levels, independent of age and gestational week, revealed that MPV was significantly higher in the Group 3 than in the Groups 1 and 2, whereas $\mathrm{HbAlc}$ was significantly higher in Group 1 than in Groups 2 and $3(\mathrm{p}<0.05)$ (Table 4).

\begin{tabular}{|c|c|c|c|c|}
\hline & Group 1 & Group 2 & Group 3 & \\
\hline & Mean $\pm s . d$ & Mean \pm s.d & Mean \pm s.d & $p$ \\
\hline $\mathbf{H b}$ & $11.55 \pm 0.93$ & $11.43 \pm 0.90$ & $11.62 \pm 0.77$ & 0.831 \\
\hline MPV & $8.53 \pm 0.99$ & $8.35 \pm 1.34$ & $9.43 \pm 1.09$ & 0.000 \\
\hline HbA1c & $4.94 \pm 0.26$ & $5.17 \pm 0.65$ & $5.02 \pm 0.52$ & 0.000 \\
\hline Fasting plasma glucose & $81.74 \pm 9.27$ & $86.09 \pm 7.87$ & $92.47 \pm 11.45$ & 0.000 \\
\hline
\end{tabular}

Gestational diabetes risk was observed to rise 1.155 -fold (1.090-1.224) for each one year increase in age, 2.098-fold (1.586-2.776) for each unit of increase in MPV, 3.509-fold (1.371-8.892) for each unit of increase in HbA1c, and 1,098fold (1.064-1.134) for each unit of increase in FBG (Table 5).

In the ROC analysis, the area under the curve exhibited significant results at all MPV cut-off values within 8-10 fL range. The highest area under the curve was determined at 8.5-8 fL range. The MPV cut-off value of $>8.8 \mathrm{fL}$ showed the highest area under the curve.

Table 4. Comparison of the groups with regard to MPV, HbA1c, and fasting plasma glucose levels, independent of age and gestational week

\begin{tabular}{lccccccccccc} 
& \multicolumn{3}{c}{ Group 1 } & \multicolumn{3}{c}{ Group 2 } & \multicolumn{3}{c}{ Group 3 } \\
\hline & Mean & Lowest & Highest & Mean & Lowest & Highest & Mean & Lowest & Mean & $\boldsymbol{p}$ \\
\hline MPV & 8.5 & 8.4 & 8.7 & 8.4 & 8.0 & 8.8 & 9.4 & 9.2 & 9.7 & 0.000 \\
HbA1c & 4.8 & 4.7 & 4.8 & 5.0 & 4.9 & 5.2 & 5.0 & 4.9 & 5.1 & 0000 \\
Fasting plasma glucose & 82.1 & 80.8 & 83.4 & 85.5 & 81.5 & 89.5 & 91.4 & 88.9 & 94.0 & 0.000 \\
\hline
\end{tabular}

Mancova (LCD), MPV: Mean platelet volume; HbA1c: Hemoglobin A1c 


\begin{tabular}{lcccc} 
Table 5. The effects of age, MPV, & HbA \\
glucose levels on GDM development & and fasting plasma \\
& \multicolumn{5}{c}{$\begin{array}{c}\text { \%5 Confidence } \\
\text { interval }\end{array}$} \\
\hline & OR & Lowest & Highest & $\boldsymbol{p}$ \\
\hline Age & 1.155 & 1.090 & 1.224 & 0.000 \\
MPV & 2.098 & 1.586 & 2.776 & 0.000 \\
HbA1c & 3.509 & 1.371 & 8.982 & 0.009 \\
Fasting plasma glucose & 1.098 & 1.064 & 1.134 & 0.000
\end{tabular}

MPV: Mean platelet volume; HbA1c: Hemoglobin A1c;

GDM: Gestational diabetes mellitus

The specificity and negative predictive value of mean platelet volume (MPV) values above $8.8 \mathrm{fL}$ for GDM detection were $75.9 \%$ and $38.9 \%$, respectively (Table 6).

\begin{tabular}{|c|c|c|c|c|}
\hline & & & \multicolumn{2}{|c|}{ Gestational DM } \\
\hline & & & - & + \\
\hline \multirow{6}{*}{ MPV } & & $\mathbf{N}$ & 173 & 14 \\
\hline & $\leq 8.8$ & $\%$ (in total) & $92.5 \%$ & $7.5 \%$ \\
\hline & & \% (in GDM) & & $24.1 \%$ \\
\hline & \multirow{3}{*}{$>8.8$} & $\mathbf{N}$ & 69 & 44 \\
\hline & & $\%$ (in total) & $61.1 \%$ & $38.9 \%$ \\
\hline & & \% (in GDM) & & $75.9 \%$ \\
\hline
\end{tabular}

MPV: Mean platelet volume; GDM: Gestational diabetes mellitus

\section{Discussion}

In this study comparing pregnant women with and without GDM, the MPV values were found to be significantly higher in the GDM group than in the IGT and control groups. There was no significant difference between the IGT and control groups with regard to MPV value. Each unit of increase in MPV was observed to raise the risk of GDM by 2.098 -fold (1.586-2.776).

Platelet volume is an indicator of platelet function and activation, and it is evaluated by MPV measurement (Sharp and Trinick, 1993; Demirtunç et al., 2009). Previous studies have shown higher MPV levels in non-pregnant Type II diabetic patients, as compared to the general population(Jirapinyo et al., 1993; Piazze et al., 2006). The risk factors and development of GDM appear to be similar to those of Type II DM. As in Type II DM patients, GDM patients may exhibit significant changes in MPV and hemoglobin A1c (HbAlc) values (Akyol et al., 2008). Compared to the platelets with normal volume, large platelets appear to show higher level of metabolic activity. Therefore, studies have shown raised TxA2 and low prostacyclin (PG12) levels, leading to vasoconstriction in both maternal and fetal circulation (Çoban et al., 2006; Crowther et al., 2005) Elevated MPV is recognized as an important marker of raised blood sugar level in GDM patients and it is held responsible for the microvascular and macrovascular complications.

In this study, HbAlc and fasting plasma glucose levels were significantly higher in the patients diagnosed with GDM and IGT than in the controls, and MPV demonstrated no significant correlation with $\mathrm{HbAlc}$ and FBG. HbAlc and FBG levels were lower than those noted in the Type II DM diagnostic criteria in the patients with GDM and IGT, however, the differences were statistically significant as compared to the control group. The patients with Type II DM had higher levels of FBG as compared to the patients with GDM and IGT, which may be the reason why MPV did not exhibit a significant correlation with $\mathrm{HbA1c}$ and FBG. The rarity of vascular complications in the GDM patients as compared to the Type II DM patients may be associated with this.

In our study, the optimal cut-off MPV value for GDM prediction was $>8.8 \mathrm{fL}$. The specificity and negative predictive value of MPV at $>8,8 \mathrm{fL}$ were $75.9 \%$ and $38.9 \%$, respectively. Jirapinyo et al. conducted a study on 396 women with high-risk pregnancy and the sensitivity and specificity of the 50g OGT test for GDM detection were found to be $85.7 \%$ and $65 \%$, respectively (Jirapinyo et al., 1993). More detailed studies including larger samples may be performed to compare the efficacy of MPV and 50g OGT in GDM detection, and to further evaluate the utility of MPV as a GDM screening test.

Since MPV is a marker of platelet activation, elevated MPV levels may help us in predicting secondary vascular complications.

Pregnant women with GDM are at high risk for development of hypertension and pre-eclampsia (Yogev and Visser, 2009). Dundar et al. performed a study on 1336 pregnant women and found that the sensitivity and specificity of MPV at $>8.5 \mathrm{fL}$ was $78 \%$ and $86 \%$, respectively (Dundar et al., 2008) In the study of Piazze et al. including 61 women with pregnancy-related hypertension and 122 controls, MPV was observed to be significantly higher in the hypertension group (Piazze et al., 2006). Future studies of similar design including GDM patients may be beneficial in predicting pre-eclampsia.

Gestational diabetes mellitus is a metabolic disorder characterized with varying degrees of carbohydrate intolerance. The patients with GDM diagnosis should be followed closely during the antenatal period with regard to maternal and fetal complications that may arise due to hyperglycemia. The assessment of platelet functions is increasingly used in predicting these complications. We believe that future randomized studies will be helpful in developing a MPV-related diagnostic test, adjusting the treatment, predicting the complications, and taking preventive measures in GDM patients.

\section{REFERENCES}

Bath, P.M., Butterworth, R.J., 1996. Platelet size: Measurement, physiology and vascular disease. Blood Coagul. Fibrin. 7, 157-161.

Crowther, C.A., Hiller, J.E., Moss, J.R., McPhee, A.J., Jeffries, W.S., Robinson, J.S., 2005. Effect of treatment of gestational diabetes mellitus on pregnancy outcomes. N. Engl. J. Med. 352, 2477-2486.

Demirtunç, R., Duman, D., Basar, M., Bilgin, M., Garip, T., 2009.The relation between glycemic control and trombosit activity in type 2 diabetes mellitus. J Diabetes Sci. 23, 89-94.

Dundar, O., Yoruk, P., Tutuncu, L., Erikci, A.A., Muhcu, M., Ergur A.R., Atay, V., Mungen, E., 2008. Longitudinal study of platelet size changes in gestation and predictive power of elevated MPV in development of pre-eclampsi Prenat Diagn. 28, 1052-1056. 
Çoban, E., Bostan, F., Özdoğan, M., 2006. The mean platelet volume in subjects with impaired fasting glucose. Plateletes. 17, 67-69.

Erikçi, A.A., Muhçu, M., Dündar, O., Öztürk, A., 2008. Could mean platelet volume be a predictive marker for gestational diabetes mellitus? Hematology. 131, 46-48.

Harris, S.B., Caulfield, L.E., Sugamori, M.E., Whalen, E.A., Henning, B., 1997.The epidemiology of diabetes in pregnant Native Canadians. A risk profile. Diabetes Care. 20, 422-1425.

Hekimsoy, Z., Payzin, B., Örnek, T., Kandoğan, G., 2004. Mean trombosit volume in Type 2 Diabetic patients. J. Diabetes Complications. 18, 173-176.

Jagroop, I.A., Mikhailidis, D.P., 2001. Doxazosin, an alpha1-adrenoceptor antagonist, inhibits serotonin-induced shape change in human trombosits. J. Hum. Hypertens. 15, 203-207.

Jirapinyo, M., Puavilai, G., Chanprasertyotin, S., Tangtrakul, S., 1993. Predictive value of 1 hour $50 \mathrm{~g}$ oral glucose load screening test for gestational diabetes mellitus compared pregnant women. Asia Oceania J. Obstet. Gynaecol. 19, 7-12.

Mazze, R.S., Krogh, C.L., 1992. Gestational diabetes mellitus: Now is the time for detection and treatment. Mayo. Clin. Proc. 67, 995-1002.

Papanas, N., Symeonidis, G., Maltezos, E., Mavridis, G., Karavageli, E.,Vosnakidis, T., Lakasas, G., 2004.Mean trombosit volume in patients with type 2 diabetes mellitus . Trombosits. 15, 475-478.

Park, Y., Schoene, N., Haris, W., 2002. Mean trombosit volume as an indicator of trombosit activation: Methodological issues. Trombosits. 13, 301-306.

Piazze, J., Gioia, S., Maranghi, L., Anceschi, M., 2006.Mean platelet and red blood cell volume measurements to estimate the severity of hypertension in pregnancy. J Perinat. Med. 343, 246-7.

Sharp, P.C., Trinick T., 1993. Mean trombosit volume in diabetes mellitus. Q.J.M. 86, 739-742.

Yogev, Y., Visser, G.H., 2009. Obesity, gestational diabetes and pregnancy outcome. Semin Fetal Neonatal Med. 14, 77-84. 